\title{
Type I parametric down conversion of highly focused Gaussian beams in finite length crystals
}

\author{
Yasser Jeronimo-Moreno and R. Jáuregui \\ Instituto de Física, Universidad Nacional Autónoma de México, \\ Apartado Postal 20-364, México DF 01000, México \\ *Corresponding author: yjeronimo@fisica.unam.mx,rocio@fisica.unam.mx
}

\begin{abstract}
We study the correlations in wave vector space of photon pairs generated by type I spontaneous parametric down conversion using a Gaussian pump beam. We analyze both moderate focused and highly focused regimes taking special attention to the angular spectrum and the conditional angular spectrum. Simple analytic expressions are derived that allow us to study in detail the dependence of these spectra on the waist of the source and the length of the nonlinear crystal. These expressions are in good agreement with numerical expectations and reported experimental results. They are used to make a systematic search of optimization parameters that improve the feasibility of using highly focused Gaussian beams to generate idler and signal photons with predetermined mean values and spread of their transverse wave vectors.
\end{abstract}

PACS numbers: 42.50.-p, 42.65.Lm

\section{INTRODUCTION}

The process of spontaneous parametric down conversion (SPDC) 1], where a pump beam interacts in a non linear crystal and individual photons of the pump decay in a pair of photons, is one of the most reliable sources of photons with predetermined properties 2. In particular, the two photon state may exhibit spatial entanglement [3] 5] that can be manipulated via the modification of the spatial structure of the pump beam and/or the crystal length. Varying them, it is even possible to optimize the coupling efficiency to other optical elements like optical fibers [6-12.

Expressions for the main properties of the emission cone and their dependence on the crystal length have been the subject of intense research because of their potential use in the quantum engineering of SPDC [3, 13[19. In this work, we study the spatial properties of the emitted photons via the angular spectrum (AS) and the conditional angular spectrum (CAS). Using the exact expressions for the birefringent dispersion relations, we numerically evaluate these spectra both in the moderate focused and highly focused regimes of a Gaussian pump beam. When reliable approximations for the phase matching function are used, we also obtain simple analytical expressions that reproduce accurately the numerical expectations and reported experimental results; they allow a better understanding of the effects of the pump beam waist and crystal length in both the AS and CAS functions. We also study the usage of these expressions for the optimization of the SPDC process in the generation of idler and signal photons localized in predetermined regions of the wave vector space.

\section{THEORY.}

To first order in perturbation theory, the SPDC state is given by [2]:

$$
|\Psi\rangle=|\mathrm{vac}\rangle+\int d \omega_{s} d \omega_{i} d^{2} \mathbf{k}_{\perp, s} d^{2} \mathbf{k}_{\perp, i} F_{s, i}\left|\alpha_{p} ; 1_{s} ; 1_{i}\right\rangle,
$$

where $F_{s, i}=F\left(\mathbf{k}_{\perp, s}, \omega_{s} ; \mathbf{k}_{\perp, i}, \omega_{i}\right)$ represents the joint amplitude expressed as a function of the frequency $\omega_{b}$ and the transversal wave vectors $\mathbf{k}_{\perp, b}$ for the signal and idler modes $(b=s, i)$. It has the following structure:

$$
F\left(\mathbf{k}_{\perp, s}, \omega_{s} ; \mathbf{k}_{\perp, i}, \omega_{i}\right)=g \mathfrak{E}\left(\mathbf{k}_{\perp, p}, \omega_{p}\right) f\left(\mathbf{k}_{\perp, s}, \omega_{s} ; \mathbf{k}_{\perp, i}, \omega_{i}\right)
$$

with $g$ the product of the effective second order susceptibility and the normalization factors of the quantized pump, signal and idler photon states. $\mathfrak{E}$ is the amplitude function of a Gaussian coherent pump beam,

$$
\begin{aligned}
\mathfrak{E}\left(\mathrm{k}_{x, p}, \mathrm{k}_{y, p}, \omega_{p}\right) & =\alpha\left(\omega_{p}\right) \cdot \mathrm{e}^{-\left(k_{x, p} W_{x} / 2\right)^{2}\left(1-i \frac{z_{o}}{z_{R x}}\right)} \\
& \times \mathrm{e}^{-\left(k_{y, p} W_{y} / 2\right)^{2}\left(1-i \frac{z_{o}}{z_{R y}}\right)}
\end{aligned}
$$

with $\alpha\left(\omega_{p}\right)$ its spectral amplitude, $W_{x}$ and $W_{y}$ the values of waist pump along $x$-axis and $y$-axis respectively, $z_{o}$ determines the focal plane and $z_{R}$ are the Rayleigh lengths along $x$-axis and $y$-axis. Energy conservation implies $\omega_{p}=\omega_{s}+\omega_{i}$. For a wide crystal, the conservation of transversal momenta yields the condition $\mathbf{k}_{\perp, p}=\mathbf{k}_{\perp, i}+\mathbf{k}_{\perp, s}$. In Eq. (2), $f$ corresponds to the phase matching function:

$$
f\left(\mathbf{k}_{\perp, s}, \omega_{s} ; \mathbf{k}_{\perp, i}, \omega_{i}\right)=L \operatorname{sinc}\left(L \Delta \mathrm{k}_{z} / 2\right) \mathrm{e}^{i L \Delta \mathrm{k}_{z} / 2},
$$

where $L$ is the crystal length and the mismatch term is $\Delta k_{z}=k_{z, p}-k_{z, s}-k_{z, i}$.

In this paper we concentrate on type-I SPDC and degenerated emission, i.e., $\omega_{p}=\omega=2 \omega_{s}=2 \omega_{i}$. Thus, the 
emitted photons have ordinary polarization and their dispersion relation is

$$
\mathrm{k}_{z, b}^{O}=\sqrt{\epsilon_{\perp} \omega^{2} / 4 c^{2}-k_{\perp, b}^{2}} \quad b=i, s
$$

with $c$ the velocity of light in vacuum, $\epsilon_{\perp}$ is the permeability function transversal to the ordinary plane defined by the optical axis $\mathbf{a}=\left(0, \mathrm{a}_{y}, \mathrm{a}_{\mathrm{z}}\right)$; the ordinary refraction index is $n_{o}=\sqrt{\epsilon_{\perp}}$. The permeability coefficient parallel to the optical axis $\epsilon_{\|}$yields the dispersion relation for the pump wave which evolves in the extraordinary plane:

$$
\begin{aligned}
\mathrm{k}_{z}^{E}\left(\mathbf{k}_{\perp}, \omega\right) & =-\beta \mathbf{a} \cdot \mathbf{k}_{\perp}+\frac{\omega}{c} \mathrm{n}_{e f f} \sqrt{1-\frac{k_{\perp}^{2} c^{2}}{\omega^{2}} \eta}, \\
\mathrm{n}_{e f f} & =\sqrt{\frac{\epsilon_{\perp} \epsilon_{\|}}{\epsilon_{\perp}+\Delta \epsilon \mathrm{a}_{z}^{2}}}, \quad \beta=\frac{\Delta \epsilon \mathrm{a}_{z}}{\epsilon_{\perp}+\Delta \epsilon \mathrm{a}_{z}^{2}}, \\
\eta & =\frac{1}{\epsilon_{\perp}+\Delta \epsilon \mathrm{a}_{z}^{2}},
\end{aligned}
$$

$\Delta \epsilon=\epsilon_{\|}-\epsilon_{\perp}$. The $\beta$ term is responsible for the deviation of the Poynting vector with respect to the pump wavefront inside the crystal, the so called walk off effect 22 25]. The $\eta$ term gives rise to astigmatic effects [24]. In the limit of normal incidence, this equation reduces to the expression of the effective refractive index experienced by a paraxial pumping extraordinary wave, $\mathrm{k}_{z}^{E}\left(\mathbf{k}_{\perp} \sim \mathbf{0}\right)=$ $\mathrm{n}_{\text {eff }} \omega / c$. The permeability coefficients $\epsilon_{\|}$and $\epsilon_{\perp}$ depend on the light frequency and some experimental set ups are implemented assuming $n_{\text {eff }}(\lambda)=n_{o}(2 \lambda)$. We shall consider the general case where this equation is not taken for guaranteed.

The distribution of the signal/idler photons in the wave vector domain is the angular spectrum (AS):

$$
R_{s}\left(\mathbf{k}_{s}, \omega_{s} ; \omega_{i}\right)=\int d \mathbf{k}_{\perp, i}\left|F\left(\mathbf{k}_{\perp, s}, \omega_{s} ; \mathbf{k}_{\perp, i}, \omega_{i}\right)\right|^{2}
$$

The conditional angular spectrum (CAS), which is a function of $\mathbf{k}_{\perp, s}$ and $\mathbf{k}_{\perp, i}$, is defined as:

$$
R_{c}\left(\mathbf{k}_{\perp, s}, \omega_{s} ; \mathbf{k}_{\perp, i}, \omega_{i}\right)=\left|F\left(\mathbf{k}_{\perp, s}, \omega_{s} ; \mathbf{k}_{\perp, i}, \omega_{i}\right)\right|^{2}
$$

This function represents the probability to detect a signal photon with wave vector $\mathbf{k}_{\perp, s}$ in coincidence with an idler photon with wave vector $\mathbf{k}_{\perp, i}$. In the paraxial regime $\left(W k_{z, p} \gg 1\right)$ the pump beam can be approximated by a plane wave, and, if we consider a long crystal, the SPDC process results in a strict momentum conservation that correlates the observation of an individual signal photon with $\mathbf{k}$-vector to a single idler photon with a well defined $\mathbf{k}$-vector. In experimental situations involving the small but finite transverse dimensions of the pump beam and a usually wide but not so long crystal, there is a set of relevant pump wave vectors $\left\{\mathbf{k}_{p}\right\}$ that are close to satisfy the phase matching condition $\Delta \mathbf{k}_{z}=0$ for given idler and signal wave vectors $\mathbf{k}_{i, s}$. Notice that $R_{c}$ results independent of the phase factor which contains the information about the Rayleigh lengths. That is, $R_{c}$ depends just on the amplitude of the Fourier content of the pump beam.
In order to obtain approximate expressions for the AS and CAS functions, we make a first order Taylor description of the phase mismatch,

$$
\begin{aligned}
\Delta k_{z} & \sim \kappa-\mathbf{d} \cdot\left(\mathbf{k}_{\perp, s}+\mathbf{k}_{\perp, i}\right), \\
\kappa & =(\omega / c)\left(n_{e f f}-n_{o}\right)+\left(2 c / n_{o} \omega\right) k_{\perp, s}^{2}, \\
\mathbf{d} & =\beta \mathbf{a}+\left(2 c / n_{o} \omega\right) \mathbf{k}_{\perp, s} .
\end{aligned}
$$

Note that in the absence of walk off effects $\mathbf{d}$ would point in the $\mathbf{k}_{\perp, s}$ direction. Approximating the function $\operatorname{sinc}(x)$ by a Gaussian function $\exp \left[-(\gamma x)^{2}\right], \gamma=0.4393$, the expression for the CAS becomes:

$$
\begin{aligned}
& R_{c}\left(\mathbf{k}_{\perp, s}, \omega_{s} ; \mathbf{k}_{\perp, i}, \omega_{i}\right)=\left|g L \alpha\left(\omega_{s}+\omega_{i}\right)\right|^{2} \times \\
& \mathrm{e}^{-\sigma_{x}^{2}\left(k_{x, s}+k_{x, i}\right)^{2}-\sigma_{y}^{2}\left(k_{y, s}+k_{y, i}\right)^{2}} \mathrm{e}^{-\gamma^{2} L^{2}\left(\kappa^{2}-2 \kappa \mathbf{d} \cdot\left(\mathbf{k}_{\perp, s}+\mathbf{k}_{\perp, i}\right)\right) / 2} \\
& \mathrm{e}^{-\left(\gamma^{2} L^{2} / 2\right)\left(2 d_{x} d_{y}\left(k_{x, s}+k_{x, i}\right)\left(k_{y, s}+k_{y, i}\right)\right)}
\end{aligned}
$$

$\sigma_{x}^{2}=\left(W_{x}^{2}+\gamma^{2} L^{2} d_{x}^{2}\right) / 2, \quad \sigma_{y}^{2}=\left(W_{y}^{2}+\gamma^{2} L^{2} d_{y}^{2}\right) / 2$.

The effective width of the Gaussian factor that modulates the CAS function has two contributions, one due solely to the pump geometry and another highly dependent on the crystal optics and geometry. The latter includes a term $\sim L\left(\Delta \epsilon \mathrm{a}_{z} \mathbf{a} /\left(\epsilon_{\perp}+\Delta \epsilon \mathrm{a}_{z}^{2}\right)\right)$. In the paraxial regime the CAS function will be determined by the geometry of the Gaussian pump beam whenever $W_{b} \gg L\left(\Delta \epsilon \mathrm{a}_{z} \mathrm{a}_{b} /\left(\epsilon_{\perp}+\Delta \epsilon \mathrm{a}_{z}^{2}\right)\right)$. In fact, for long crystals, second order terms proportional to $k_{\perp, p}^{2}$ in the extraordinary dispersion relation, Eq. (6), could become relevant. In such a case a third term contributes to the effective width of the Gaussian pump beam:

$$
\tilde{W}_{b}^{2}=W_{b}^{2}+\frac{\gamma^{2} L^{2} n_{o}^{2}}{\epsilon_{\perp}+\Delta \epsilon \mathrm{a}_{z}^{2}}\left(\frac{n_{e f f}}{n_{o}}-\frac{n_{e f f}^{2}}{n_{o}^{2}}\right), \quad b=x, y
$$

replaces $W_{b}^{2}$ in Eq. (14). In general, for a symmetric pump beam $W_{x}=W_{y}=W$, the CAS function may exhibit an asymmetric profile due to the orientation of the birefringent axis as encoded in $\mathbf{d}$.

We shall now illustrate the CAS function by (i) choosing the value of the transversal wave vector of the idler photon $\mathbf{k}_{\perp, i}^{0}$ that maximizes the counts and (ii) reporting the corresponding distribution of the signal transverse wave vector, $\mathbf{k}_{\perp, s}$. We take parameters from particular reported experimental situations 19. This allows to confirm the reliability of our numerical simulations. Thus, we consider a $1 \mathrm{~mm}$ length BBO crystal, cut for type-I phase matching for degenerated emission with angle $\theta_{\mathbf{a}}=29.3^{\circ}$, (the optical axis is defined by $\left.\mathbf{a}=\left(0, \sin \theta_{\mathbf{a}}, \cos \theta_{\mathbf{a}}\right)\right)$; the quasi monochromatic pump beam has a wavelength centered at $406.99 \mathrm{~nm}$. Calculations are performed both using the exact expressions for the birefringent dispersion relations for the ordinary wave, Eq. (5), and extraordinary wave, Eq.66), as well as using the approximate analytical equation (14).

Fig.1a(A) shows the numerical CAS function for a symmetric pump waist $W_{x}=W_{y}=W=185 \mu \mathrm{m}$. As expected from Eq. (14), for a pump waist that validates the paraxial regime and $W \gg \gamma L|\mathbf{d}|$, the CAS describes highly 
localized momentum correlations determined mainly by the pump waist $W$. Fig.1a(B) illustrates the CAS function calculated from the approximate Eq.(14). In order to make a more clear comparison between numeric and analytic calculations, Figs 1a(C)-(D) show the marginal distributions $\mathcal{M}\left(k_{b, s}\right)=\int d k_{j \neq b, s} R_{c}\left(\mathbf{k}_{\perp, s}, \omega_{s} ; \mathbf{k}_{\perp, i}^{0}, \omega_{i}^{0}\right)$ for $b, j=x, y$. The resulting agreement between the numerical and analytic calculations is also very good.

In Fig $1 \mathrm{~b}$ (A) the CAS is illustrated for a symmetric focused pump beam $W=35 \mu \mathrm{m}$. Notice that the spread of the marginal distributions is, in this case, about twice that observed for a pump beam with $W=185 \mu \mathrm{m}$. This is a direct consequence of the fact that a higher focusing necessarily involves the incorporation of a wider distribution of pump wave vectors.

For a highly focused beam, as that shown in Fig. 1c where $W=5 \mu \mathrm{m}$, the CAS function profile spreads even more and the conditional angular spectra becomes more sensitive to the crystal length and optical parameters as described by the analytical expression, Eq. (14), Notice that although this approximate distribution does not reproduce in all details its numeric analog, it has the correct central point and width. The discrepancy is mainly due to the usage of the approximate dispersion relation as described above. Another effect of the crystal is present in the small oscillations in the marginal distribution $\mathcal{M}\left(k_{y, s}\right)$ in Fig $1 \mathrm{c}$ (D) that were lost in the replacement of the phase matching sinc by a Gauss function. Besides, for this highly focused pump beam there is an observable difference between the absolute mean values of the $\mathrm{y}$-component of the wave vector of the idler photon $k_{y, i}=-0.51 \mu \mathrm{m}^{-1}$ and of the signal photon $\left\langle k_{y, s}\right\rangle \sim 0.57 \mu \mathrm{m}^{-1}$. The CAS for $W=185 \mu \mathrm{m}$ and $W=35 \mu \mathrm{m}$ can be successfully compared with Fig. 2 of Ref. [19].

In order to illustrate other interesting features of the CAS, we now consider the particular case in which the condition $k_{x, s}=k_{x, i}=0$ is imposed in the idler and signal photons and compare it to the case $k_{y, s}=k_{y, i}=0$. This results in a conditional distribution for $k_{y, s}$ and $k_{y, i}$, and a conditional distribution for $k_{x, s}$ and $k_{x, i}$ respectively. The CAS's are illustrated in Fig. 2 taking similar parameters as those used in previous examples. For $W=185 \mu \mathrm{m}$ the signal and idler transverse wave vectors are restricted to almost opposite directions, so that for $k_{x, s}=k_{x, i}=0, k_{y, s} \sim-k_{y, i}$ with $\left|k_{y, i}\right| \sim 0.5 \mu \mathrm{m}^{-1}$. As the pump beam waist decreases, the anisotropic role of higher values of $\mathbf{k}_{\perp, p}$ in the phase matching condition is more relevant. These has two consequences. One of them is the increasing width on the CAS distributions. The other is that such distributions become clearly different for the condition $k_{x, s}=k_{x, i}=0$ and the condition $k_{y, s}=k_{y, i}=0$.

An approximate closed expression for the angular spectra distribution can also be obtained. For simplicity we consider a symmetric pump profile $\left(W_{x}=W_{y}=W\right)$. Using the conservation of transverse momentum, writing the pump integration variable $\mathbf{k}_{\perp, p}$ in polar coordinates, and performing a rotation of the integration variable by an angle $\theta_{r}=\arccos \left(d_{x} /|\mathbf{d}|\right)$, one obtains:

$$
\begin{gathered}
R_{s}\left(\mathbf{k}_{s}\right)=|g L \alpha|^{2} \mathrm{e}^{-\sigma_{\mathrm{AS}}^{-2}\left(k_{\perp, s}^{2}-r_{\mathrm{AS}}^{2}\right)^{2}} \int_{0}^{2 \pi} d \theta \int_{0}^{\tilde{k}_{\perp}} d k_{\perp} k_{\perp} \mathrm{e}^{-u} \\
r_{\mathrm{AS}}^{2}=\frac{1}{2}\left(\frac{n_{o} \omega}{c}\right)^{2}\left(1-\frac{n_{e f f}}{n_{o}}\right), \sigma_{\mathrm{AS}}^{-2}=\frac{2\left(\gamma L c / n_{o} \omega\right)^{2}}{1+(\gamma L|\mathbf{d}| / W)^{2}} \\
\tilde{k}_{\perp}=\frac{\omega \sqrt{\epsilon_{\perp} \epsilon_{\|}}}{c \sqrt{\epsilon_{\perp}+\Delta \epsilon \mathrm{a}_{\perp}^{2} \cos ^{2} \theta}}, \zeta_{x}=\frac{\kappa|\mathbf{d}|}{|\mathbf{d}|^{2}+(W / \gamma L)^{2}}(16)
\end{gathered}
$$

$u=\left(\left(W^{2}+(\gamma L|\mathbf{d}|)^{2}\right)\left(k_{\perp} \cos \theta-\zeta_{x}\right)^{2}+W^{2} k_{\perp}^{2} \sin ^{2} \theta\right) / 2$. By evaluating $\partial u\left(k_{\perp}, \theta\right) / \partial k_{\perp}$ it can be shown that

$$
\begin{gathered}
R_{s}\left(\mathbf{k}_{\mathbf{s} \mathbf{0}}\right)=\left|g L \alpha\left(\omega_{p}\right)\right|^{2} \mathrm{e}^{-\sigma_{\mathrm{AS}}^{-2}\left(k_{\perp, s}^{2}-r_{\mathrm{AS}}^{2}\right)^{2}}\left[\int_{0}^{2 \pi} d \theta \frac{1-\mathrm{e}^{-u}}{\operatorname{den}(\theta)}\right. \\
\left.+W \zeta_{x} \int_{0}^{2 \pi} \frac{d \theta \cos \theta}{\operatorname{den}(\theta)} \int_{0}^{\tilde{\kappa}_{\perp}} d k_{\perp} \mathrm{e}^{-u}\right],
\end{gathered}
$$

$\operatorname{den}(\theta)=\left(W^{2}+(\gamma L|\mathbf{d}|)^{2}\right) \cos ^{2} \theta+W^{2} \sin ^{2} \theta$. The second integral over the variable $d k_{\perp}$ can be expressed in terms of error functions, and the remaining integral over the variable $\theta$ could be evaluated numerically. Alternatively we can also make an approximate evaluation of Eq. (17). At the angle $\theta_{\text {est }}=\arccos \left(\kappa /|\mathbf{d}| k_{\perp, s} c\right)$ the exponent $u\left(k_{\perp}, \theta\right)$ takes an stationary value: $\partial u\left(k_{\perp}, \theta_{e s t}\right) / \partial \theta=0$. We replace $u\left(k_{\perp}, \theta\right)$ by $u\left(k_{\perp}, \theta_{\text {est }}\right)=\frac{W^{2}}{2} k_{\perp}^{2}-\frac{\gamma^{2} L^{2} \kappa^{2}}{2}[1-$ $\left.\frac{1}{1+(W / \gamma L|\mathbf{d}|)^{2}}\right]$ in Eq. $\sqrt{17}$. Performing the remaining integrals we obtain:

$$
R_{s}\left(\mathbf{k}_{\mathbf{s} \mathbf{0}}\right) \approx \frac{\pi\left|g L \alpha\left(\omega_{p}\right)\right|^{2} \mathrm{e}^{-\sigma_{\mathrm{AS}}^{-2}\left(k_{\perp, s}^{2}-r_{\mathrm{AS}}^{2}\right)^{2}}}{W^{2} \sqrt{1+(\gamma L|\mathbf{d}| / W)^{2}}}\left(1-e^{-u\left(\tilde{k}_{\perp}, \theta_{e s t}\right)}\right) .
$$

In most practical situations $1 \gg e^{-u\left(\tilde{k}_{\perp}, \theta_{e s t}\right)}$.

We can now interpret the different terms appearing in Eq. 18. For a not too long crystal $u\left(\tilde{k}_{\perp}, \theta_{\text {est }}\right) \sim$ $-W^{2} \tilde{k}_{\perp}^{2} / 2$ which does not depend on $k_{\perp, s}$; in this case $1 \gg e^{-u\left(\tilde{k}_{\perp}, \theta_{e s t}\right)}$ just requires $W \gg \tilde{k}_{\perp}^{-1}$. Under these conditions the AS describes a conical emission with radius $r_{A S}$ specified just by the difference of the refractive indices. If $n_{e f f}(\lambda)=n_{o}(2 \lambda), r_{A S}=0$ corresponding to the collinear regime. For the particular SPDC source described above $r_{A S}=0.49 \mu \mathrm{m}^{-1}$. The width of the emission cone $\sigma_{\mathrm{AS}}$ depends on the value of the pump waist $W$ and the crystal length $L$. In Fig $3 \mathrm{a}$ (A) we illustrate the AS for the paraxial regime, $W=185 \mu \mathrm{m}$ and $L=1 \mathrm{~mm}$ and the predicted width of the cone is $\sigma_{\mathrm{AS}}=0.03 \mu \mathrm{m}^{-1}$ which coincides, within the given significant figures, with the results obtained numerically using the exact expressions of the dispersion relations and the integrals involved in the calculations. Due to the Gaussian approximation of the sinc function used to obtain Eq. (18) this expression is unable to reproduce faint radial oscillations of the exact AS. This can be better observed in the marginal 

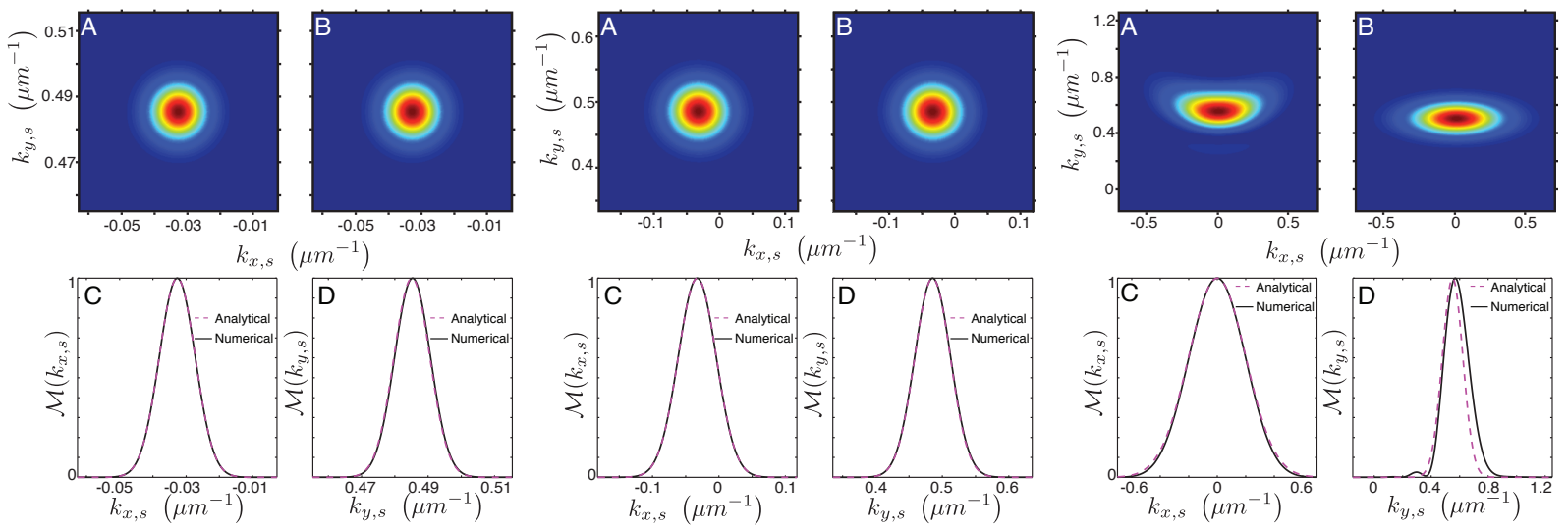

(a)

(b)

(c)

FIG. 1: Conditional angular spectra (CAS) for a $1 \mathrm{~mm}$ BBO crystal using a symmetric Gaussian pump with waist (a) $W=185 \mu \mathrm{m} ;$ (b) $W=35 \mu \mathrm{m}$;(c) $W=5 \mu \mathrm{m}$. The transverse wave vectors of the corresponding idler photon are (a)

$k_{x, i}=0.033 \mu \mathrm{m}^{-1}, k_{y, i}=-0.485 \mu \mathrm{m}^{-1}$ (b) $k_{x, i}=0.033 \mu \mathrm{m}^{-1}, k_{y, i}=-0.485 \mu \mathrm{m}^{-1}$ and (c) $k_{x, i}=0.000 \mu \mathrm{m}^{-1}$, $k_{y, i}=-0.506 \mu \mathrm{m}^{-1}$. These wave vectors were chosen so that the counts are maximized. In (A) CAS is calculated numerically, and in (B) analytically. Figs. (C)-(D) are the marginal distributions calculated numerically (solid line) and analytically (dashed line).
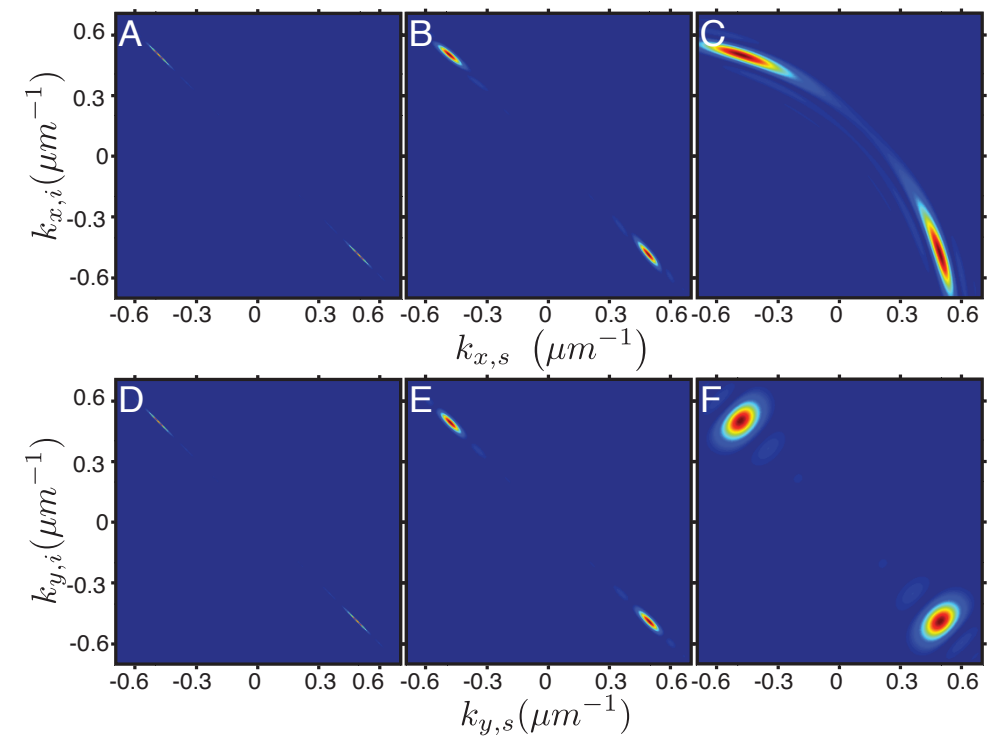

FIG. 2: In the first row the conditional spectrum that arise from demanding $k_{y, s}=k_{y, i}=0$ is illustrated for pump waists (a) $W=185 \mu \mathrm{m}$; (b) $W=35 \mu \mathrm{m}$;(c) $W=5 \mu \mathrm{m}$. In the second row, the conditional spectrum that arises from demanding $k_{x, s}=k_{x, i}=0$ is illustrated for the same pump waists. The general parameters are the same as those reported in Fig. 1 .

distribution with respect the variable $k_{x, s}$ and $k_{y, s}$ illustrated in Figs 3a (C)-(D).

We now compare the predictions of Eq. 18 for pump beams outside the paraxial regime. Fig $3 \mathrm{~b}(\mathrm{~A})$ illustrates the AS function calculated numerically using a narrower pump waist ( $W=35 \mu \mathrm{m}$ ) and the same crystal parameters described above. The pump beam focusing gives rise to an observable asymmetry in the cone width that can be attributed to an asymmetric failure of the incor- porated wave vectors $\mathbf{k}_{p}$ to fulfill the phase matching condition. In our approximate expression, this asymmetry is encoded in the factor $L|\mathbf{d}| / W$ that appears in the expression of $\sigma_{\mathrm{AS}}$. This factor cannot be neglected out of the paraxial regime even for a narrow crystal $(L=1 \mathrm{~mm}$ in Fig 3b). Fig 3b(B) illustrates the excellent agreement between the numerical and approximate results which in fact also agree with the experimental results reported in Ref. [19]. For even higher focused Gaussian beams, the 

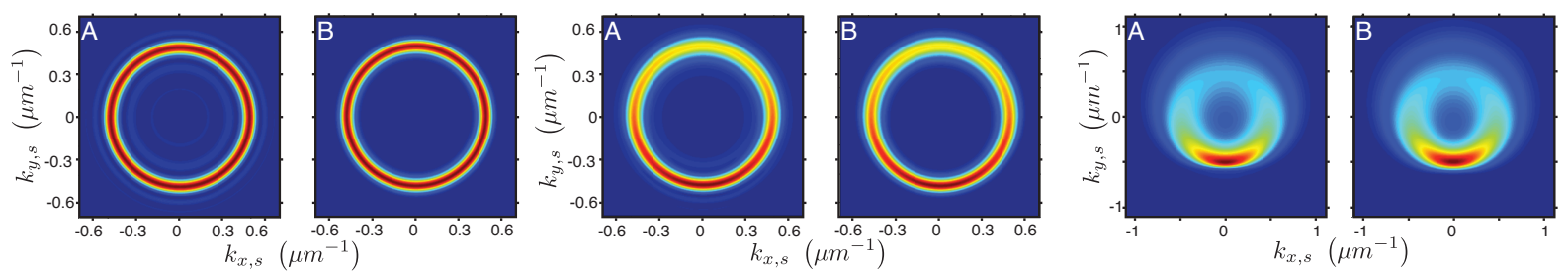

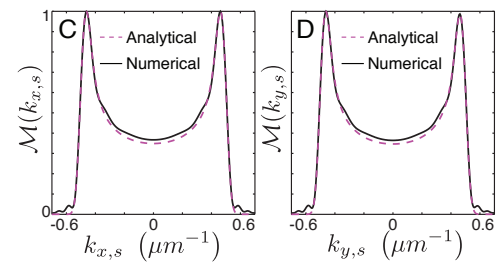

(a)

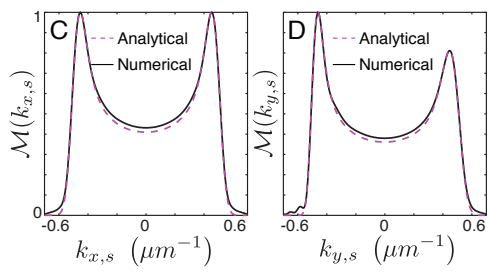

(b)

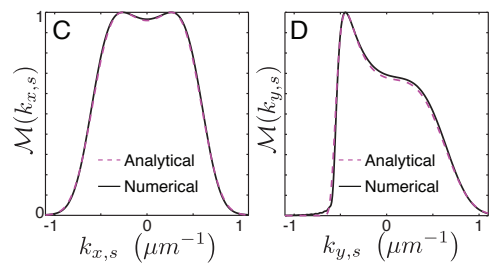

(c)

FIG. 3: Angular spectrum (AS) for a Gaussian pump with waist (a) $W=185 \mu \mathrm{m}$; (b) $W=35 \mu \mathrm{m}$;(c) $W=5 \mu \mathrm{m}$. In (A) the AS function is calculated numerically and in (B) using the approximate analytical expression. (C)-(D) are the marginal distributions obtained numerically (solid line) and analytically (dashed line).

AS function loses completely its annular symmetry as shown in Fig. $3 \mathrm{c}$ (A) where $W=5 \mu m$. The broadening in the $k_{y, s}$ direction is determined by the factor $\beta \mathrm{a}_{y}$. Even in these extreme conditions the approximate expression Eq. 18 reproduces with high accuracy the expectations from the numerical descriptions.

\section{SIGNAL AND IDLER WAVE VECTOR PROBABILITY DISTRIBUTIONS.}

From the approximate analytical expression for the angular spectrum we can study the marginal distributions on the $\mathbf{k}_{\perp, s}$ and $\mathbf{k}_{\perp, i}$ variables. Since the conditional angular spectrum shows that the idler and signal photons will be emitted nearby a cone with transversal radius $r_{A S}$, defined in Eqs. (16), it results interesting to analyze the relations between the angles of emission $\theta_{i}$ and $\theta_{s}$ of the idler and signal photons in that cone. We find easier to make such an study in terms of the mean angle, $\theta_{+}=\left(\theta_{i}+\theta_{s}\right) / 2$ and the difference angle $\theta_{-}=\theta_{s}-\theta_{i}$. The distribution

$$
\begin{gathered}
\Re_{r_{A S}}\left(\theta_{+}, \theta_{-}\right)=\left|F\left(\mathbf{k}_{\perp, s}, \omega_{s} ; \mathbf{k}_{\perp, i}, \omega_{i}\right)\right|^{2} /\left|g L \alpha\left(\omega_{p}\right)\right|^{2}, \\
\mathbf{k}_{\perp, s}=r_{A S}\left(\cos \left(\theta_{+}+\theta_{-} / 2\right), \sin \left(\theta_{+}+\theta_{-} / 2\right)\right), \\
\mathbf{k}_{\perp, i}=r_{A S}\left(\cos \left(\theta_{+}-\theta_{-} / 2\right), \sin \left(\theta_{+}-\theta_{-} / 2\right)\right),
\end{gathered}
$$

is illustrated for the pump beam waists $W=185 \mu \mathrm{m}$, $W=35 \mu \mathrm{m}$ and $W=5 \mu \mathrm{m}$ in Fig 4 , taking the same general parameters used in the simulations reported before; in particular the crystal cut angle is $\theta_{\mathbf{a}}=29.3^{\circ}$ yielding $r_{A S}^{29.3^{\circ}}=0.492 \mu \mathrm{m}^{-1}$. The exact dispersion relations for the pump, idler and signal photons were used. The calculation results coincide with those obtained using the approximate dispersion relations for $W>25 \mu \mathrm{m}$ within the first three significant figures. Notice that the idler and signal photons are emitted mainly in opposite transverse directions since the maximum value of $\mathfrak{R}_{r_{A S}}\left(\theta_{+}, \theta_{-}\right) \sim 1$ is always taken for $\theta_{-}=180^{\circ}$. However, for $W<25 \mu \mathrm{m}$ the width of the marginal distribution of $\theta_{-}$increases as the waist decreases, so that there is a high possibility that the signal and idler photons are emitted with $\theta_{-} \in$ $\left(175^{\circ}, 185^{\circ}\right)$. It can also be observed that the emission cone will have an almost isotropic distribution of photons for $W>100 \mu \mathrm{m}$ since for those values of the pump waist $\mathfrak{R}_{r_{A S}}$ shows only a slight dependence on $\theta_{+}$. For lower values of $W$, the largest values of the marginal distribution of $\theta_{+}$are achieved for $\theta_{+}=0, \pm \pi$. If $\theta_{-} \sim \pi$, this means that $\theta_{i, s} \sim \pm \pi / 2$, which is consistent with all the results for the CAS shown in last section.

The approximate analytical expression for the CAS distribution, Eq. (18), can be used to obtain approximate expressions for the mean aperture angle of the emission cone

$$
\left\langle\Theta_{A S}\right\rangle=\sin ^{-1}\left(r_{A S} / \sqrt{\left(\omega n_{o} / 2 c\right)^{2}-r_{A S}^{2}}\right) .
$$

The spread in the distribution of this angle is determined by the spread in the distribution of the cone radius $\sigma^{1 / 2} / \sqrt{2}$, given in Eq. $(16$, and it depends on the direction of the wave vectors $\mathbf{k}_{s, i}$. Its maximum (minimum) value is achieved for signal photons emitted at angles that maximize (minimize) the magnitude of the $\mathbf{d}$ vector. For a signal photon with $\left|\mathbf{k}_{s}\right| \sim r_{A S}$, these extreme values are given by $\left|\mathbf{d}_{e x t}\right| \sim\left|\beta \cos \alpha \mp\left(2 c / n_{o} \omega\right) r_{A S}\right|$. The corresponding extreme values of the aperture angle are

$$
\begin{gathered}
\Theta_{A S}^{e x t}=\left\langle\Theta_{A S}\right\rangle+\Delta \Theta_{A S}^{e x t} \\
\Delta \Theta_{A S}^{e x t} \sim \frac{\left(1+\left(\gamma L\left|\mathbf{d}_{e x t}\right| / W\right)^{2}\right)^{1 / 4}}{2^{5 / 4} \sqrt{\gamma L\left(\omega n_{o} / 2 c\right)}} .
\end{gathered}
$$


For the general parameters used up to now, $\left\langle\Theta_{A S}\right\rangle=$ $0.038 \mathrm{rad}$ and for $W=185 \mu \mathrm{m}$ the spread $\Delta \Theta_{A S}^{\max }=0.006$ $\operatorname{rad} \sim \Delta \Theta_{A S}^{\min }$, for $W=35 \mu \mathrm{m}$ the maximum spread increases to $\Theta_{A S}^{\max }=0.009 \mathrm{rad}$ and the minimum $\Theta_{A S}^{\min }=$ $0.007 \mathrm{rad}$, and for the extreme condition $W=5 \mu \mathrm{m}$, $\Delta \Theta_{A S}^{\max }=0.022 \mathrm{rad}$ and $\Delta \Theta_{A S}^{\min }=0.017 \mathrm{rad}$.

\section{SPDC OPTIMIZATION.}

It has been shown that SPDC with extremely focused Gaussian beams in general gives rise to an anisotropic distribution of photon pairs in an emission cone with a mean radius independent of the pump waist, but with a spread that may increase as the pump waist diminishes. This fact may make difficult the separation of the idler and signal effective modes. The SPDC simulations reported in previous sections were worked out with parameters chosen to optimize a type I SPDC process for an ideally incoming plane wave. In this section we show that parameters like cut angle of the nonlinear crystal or the wavelength of the pump beam can be easily optimized to increase the emission cone radius, preserve approximately the spread of the cone radius and simultaneously preserve the anisotropic localization of the idler and signal wave vectors without a significant loss in the probability of coincidence detection of the emitted photons.

In standard experimental set-ups, the crystal cut angle is chosen so that an ideal pump beam with an almost plane wave front yields the possibility of creating almost collinear photon pairs with maximum probability. By increasing the cut angle, the effective extraordinary refraction index $n_{\text {eff }}$ decreases and the mean radius of the emission cone $r_{A S}$ increases. Thus, it is reasonable to study the behavior of SPDC for higher values of $\theta_{\mathbf{a}}$ to decrease the overlap between the idler and signal effective modes. Notice however that $\mathbf{d}_{\text {ext }}$ also depends on $\theta_{\mathbf{a}}$, and we are interested in finding optimal values of $\theta_{\mathbf{a}}$ that do not increase the width of the emission cone beyond the adequate values for the apertures of usual detecting elements. In most cases, the latter include optical fibers to which the signal and idler photons are required to couple.

Using the formalism presented in last section, the SPDC process were roughly simulated and optimal angles were easily found. In Fig. (5) we report the behavior of the $\mathfrak{R}_{r_{A S}}\left(\theta_{+}, \theta_{-}\right)$and its marginals for a pump beam with $W=5 \mu \mathrm{m}$ for $\theta_{\mathbf{a}}=29.3^{\circ}, 31.0^{\circ}$ and $33.0^{\circ}$. The mean emission cone radii are $r_{A S}^{31.0^{\circ}}=0.955 \mu \mathrm{m}^{-1}$ and $r_{A S}^{33.0^{\circ}}=1.313 \mu \mathrm{m}^{-1}$, so that $\left\langle\Theta_{A S}^{31.0^{\circ}}\right\rangle=0.075 \mathrm{rad}$ and $\left\langle\Theta_{A S}^{33.0^{\circ}}\right\rangle=0.103 \mathrm{rad}$. The angular maximum and minimum widths are $\Delta \Theta_{A S}^{\max , 31.0^{\circ}}=0.024 \mathrm{rad}$ and $\Delta \Theta_{A S}^{\min , 31.0^{\circ}}=0.013 \mathrm{rad}$, and $\Delta \Theta_{A S}^{\max , 33.0^{\circ}}=0.026 \mathrm{rad}$ and $\Delta \Theta_{A S}^{\min , 33.0^{\circ}}=0.009 \mathrm{rad}$. So that, increasing slightly the cut angle $\left\langle\Theta_{A S}\right\rangle$ can take two or three times its original value while the maximum value of $\Delta \Theta_{A S}$ is modified in less than $15 \%$. Notice that the change in the maximum value of the marginal distributions evaluated at
$r_{A S}$ change in less than $15 \%$.

Taking into account those results we have performed a complete simulation of the SPDC process for the pump beam with $W=5 \mu \mathrm{m}$ and a cut angle $\theta_{\mathbf{a}}=33^{\circ}$. The corresponding AS and CAS are shown in Fig. (6). As predicted from the simulations described in the last paragraph, the spatial resolution of the idler and signal photons has greatly increased while the location where the emitted photons is still concentrated around $\theta_{s, i} \pm \pi / 2$. The numerical evaluation of the brightness calculated from the CAS function shows that it increases in a $10 \%$ for the cut angle $\theta_{\mathbf{a}}=33^{\circ}$ compared to $\theta_{\mathbf{a}}=29.3^{\circ}$. Besides the rough estimates for the cone angle and its width given in last paragraph agree approximately with the exact numerical calculation.

We have also studied the possibility of maintaining the original cut angle $\theta_{\mathbf{a}}=29.3^{\circ}$ and optimize the pump wavelength for highly focused Gaussian beams. The first calculation using the expression for $r_{A S}$ and $\mathbf{d}$ give us that changing the pump wavelength from $406.99 \mu \mathrm{m}$ to $436 \mu \mathrm{m}(483 \mu \mathrm{m})$ yields the same results than changing to $\theta_{\mathbf{a}}=31^{\circ}\left(\theta_{\mathbf{a}}=33^{\circ}\right)$.

\section{CONCLUSIONS}

In this paper we have presented a study of the dependence of the angular spectrum and the conditional angular spectrum on the waist pump and the length of a nonlinear crystal used to achieve type I SPDC. Simple analytic expressions were derived for the these spectra, Eqs. 14 16. They make evident the relevance of the factor $\gamma|\mathbf{d}| L / W$, which includes information about the walk off, to understand the general features of these spectra. Notice that under the conditions here studied the CAS and the AS are independent on the position of the focus plane of the pump beam. However it could lead to observable effects in other properties of the down converted photons like the purity of state. The analytical expressions reproduce with high accuracy the predictions of numerical methods that do not make use of the approximations neither on the dispersions relations nor on the phase matching function. Even more important, the results presented here are in excellent agreement with experimental measurements reported in the literature.

Having an analytical expression for the CAS allows the identification of explicit expressions for the mean traverse radii and the anisotropic width of the emission cone in the wave vector space. The first depends just on the linear dielectric response and the cut angle of the birefringent crystal. The anisotropy depends on the relation between the crystal length and beam waist through the factor $\gamma|\mathbf{d}| L / W$. Given a crystal length, a highly anisotropic SPDC process for extreme focused Gaussian pump beams is especially relevant since it allows the prediction of the direction at which idler and signal photons will be generated. This anisotropy has been already reported by Fedorov et al in Ref. [20, 21, where special 

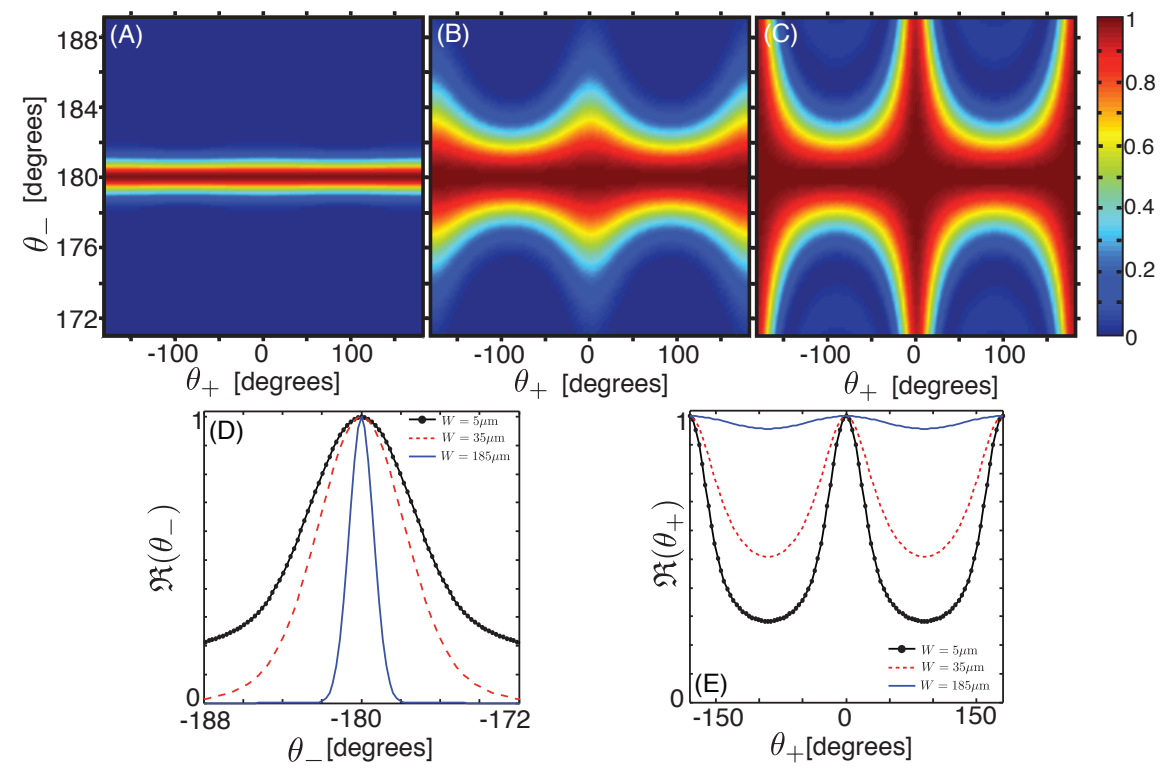

FIG. 4: Angular correlation function $\mathfrak{R}_{r_{A S}}\left(\theta_{+}, \theta_{-}\right)$for a Gaussian pump with waist (A) $W=185 \mu \mathrm{m}$;

(B) $W=35 \mu \mathrm{m} ;(\mathrm{C}) W=5 \mu \mathrm{m}$. (D)-(E) are the marginal distributions normalized to a maximum value one for the same waists of the pump beam. These distributions were obtained using the exact expressions of the dispersion relations and taking the same general parameters as in previous figures.
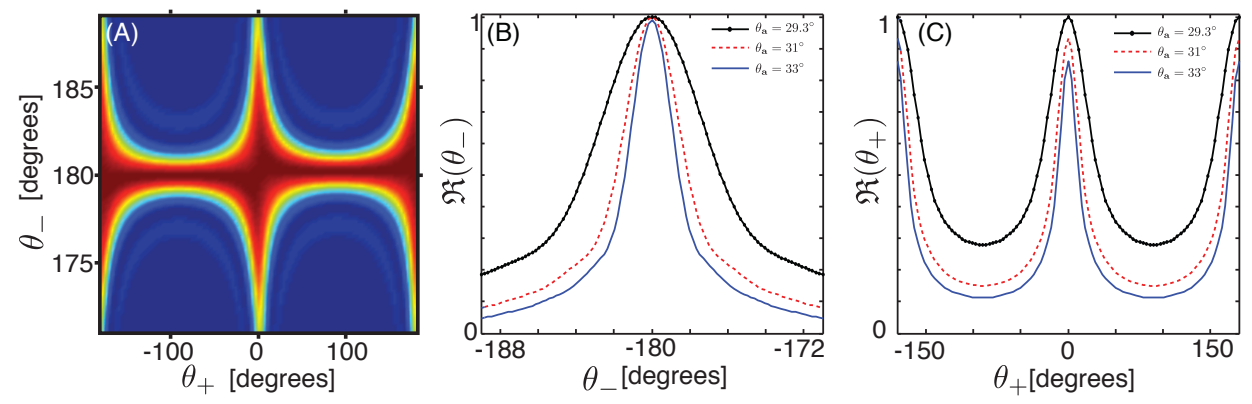

FIG. 5: Angular correlation function $\mathfrak{R}_{r_{A S}}\left(\theta_{+}, \theta_{-}\right)$and marginal distributions on the relative angle $\theta_{-}$and average angle $\theta_{+}$for a Gaussian pump waist $W=5 \mu \mathrm{m}$ and different cut angles $\theta_{\mathbf{a}}=29.3^{\circ}, \theta_{\mathbf{a}}=31.0^{\circ}$ and $\theta_{\mathbf{a}}=33.0^{\circ}$. These distributions were obtained using the exact expressions of the dispersion relations and taking the same general parameters as in previous figures.

care is taken of the theoretical and experimental analysis of the CAS and the AS along two lines one of which is contained in a plane parallel to the optical axis and the other in a plane perpendicular to that axis. In Ref. ([19]), the anisotropy is also reported in terms of the AS and the CAS, so that the comparison between their experimental and our theoretical results could be directly performed.

We have used also our expressions to make estimates of the general properties of the distribution of idler and signal photons in wave vector space. They can be used to measure entanglement [20, 21] and for an optimization of the SPDC process. That is, by simple calculations that nevertheless include the exact expressions for $\Delta k_{z}=k_{z, p}-k_{z, s}-k_{z, i}$ evaluated on $r_{A S}$ the angular distribution of the emitted photons can be studied and conditions for a given angular localization can be found; using the expression of $\sigma_{A S}$ the maximum and minimum spread the idler and signal photons may be studied looking for values that could, e. g. optimize the coupling to optical fibers without a significant loss in the probability of coincidence detection of the emitted photons. Our analysis complements the studies reported in Refs. 6 -12, providing a simple way to preselect the general experimental parameters in an optimization procedure. These parameters can be then used to make a detailed simulation of the process, previous to the experimental implementation. 


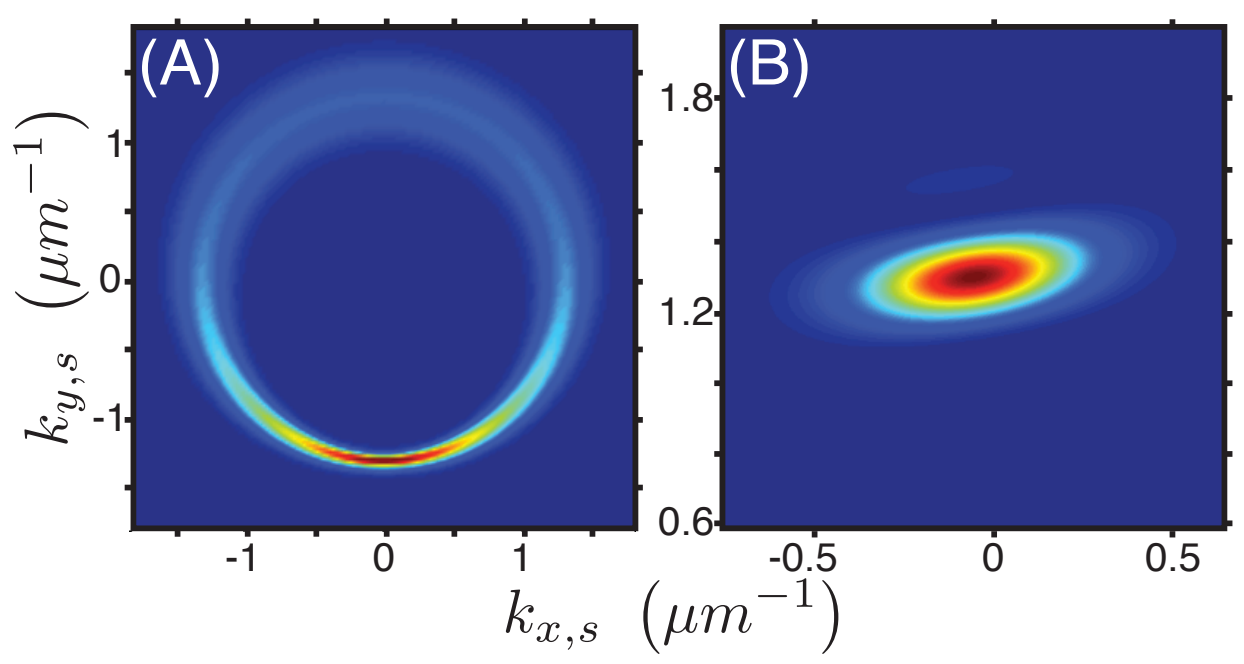

FIG. 6: (A)AS and (B) CAS for a Gaussian beam with waist $W=5 \mu \mathrm{m}$ and a cut angle of the nonlinear crystal $\theta_{\mathbf{a}}=33^{\circ}$ optimized. These distributions were obtained using the exact expressions of the dispersion relations and taking the same general parameters as in previous figures.

[1] D. C. Burnham and D. L. Weinberg, Phys. Rev. Lett. 25, 84 (1970).

[2] S. P. Walborn, C. H. Monken, S. Pádua and P. H. Souto Ribeiro, Physics Reports 495, 87 (2010).

[3] M. H. Rubin, Phys. Rev. A 54, 5349 (1996).

[4] P. G. Kwiat, J. Mod. Opt. 44, 2173 (1997).

[5] L. Neves, G. Lima, A. Delgado, and C. Saavedra, Phys. Rev. A 80, 043322(2009).

[6] F. A. Bovino, P. Varisco, A. M. Colla, G. Castagnoli, G. di Guiseppe, and A. V. Sergeinko, Opt. Commun. 227, 343 (2003).

[7] A. Dragan, Phys. Rev. A 70, 053814 (2004).

[8] R. Andrews, E. Pike, and S. Sarkar, Opt. Express 12, 3264 (2004).

[9] D. Ljunggren and M. Tengner, Phys. Rev. A 72, 062301(2005).

[10] S. Castelletto, I. Degiovanni, G. Furno, V. Schettini, A. Migdall, and M. Ware, IEEE Trans. Instrum. Meas. 54, 890 (2005).

[11] P. Kolenderski, W. Wasilewski, and K. Banaszek, Phys. Rev. A 80, 013811(2009).

[12] M. Minozzi, S. Bonora, A. V. Sergienko, G. Vallone, and P. Villoresi, Opt. Lett. 38, 489 (2013).

[13] A. U'Ren, K. Banaszek, and Walmsley, Quantum Infor. Compt. 3, 480 (2003).

[14] P. S. K. Lee, M. P. van Exter and J. P. Woerdman, Phys.
Rev. A 72, 033803 (2005).

[15] R. S. Bennink, Y. Liu, D. D. Earl, and W. P. Grice, Phys. Rev. A 74, 023802 (2006).

[16] H. Di Lorenzo Pires, F. M. G. J. Coppens, and M. P. van Exter, Phys. Rev. A 83, 033837 (2011).

[17] O. Süzer, and T. G. Goodson III, Opt. Express 16, 20166 (2008).

[18] W. P. Grice, R. S. Bennink, D. S. Goodman and A. T. Ryan, Phys. Rev. A 83, 023810 (2011).

[19] R. Ramírez-Alarcón, H. Cruz-Ramírez and A. B. U'Ren, Las. Phys. 23, 055204 (2013).

[20] M. V. Fedorov, M. A. Efremov, P. A. Volkov, E. V. Moreva,S. S. Straupe and S. P. Kulik, Phys. Rev. Lett. 99, 063901 (2007).

[21] M. V. Fedorov, M. A. Efremov, P. A. Volkov, E. V. Moreva,S. S. Straupe and S. P. Kulik, Phys. Rev. A 77, 032336 (2008).

[22] S. P. Walborn, A. N. de Oliveira, R. S. Thebaldi, and C. H. Monken, Phys. Rev. A 69, 023811 (2004).

[23] J. P. Torres, G. Molina-Terriza, and L. Torner, J. Opt. B 7, 235 (2005).

[24] A. G. da Costa Moura, W. A. T. Nogueira, S. P. Walborn and C. H. Monken, arXiv:0806.4624v1 (2008).

[25] L. E. Vicent, A. B U'Ren, R. Rangarajan, C. I. Osorio, J. P. Torres, L. Zhang and I. A. Walmsley, New J. Phys. 12, 093027 (2010). 